Turk. J. Math. Comput. Sci.

13(1)(2021) 115-121

(C) MatDer

DOI : $10.47000 /$ tjmcs. 875446

\title{
An Application to the Existence of Solutions of the Integral Equations
}

\author{
Merve Temizer Ersoy (iD \\ Department of Mathematics, Faculty of Science, Kahramanmaras Sutcu Imam University, 46100, Turkey.
}

Received: 06-02-2021 • Accepted: 23-05-2021

\begin{abstract}
Aвstract. Integral equations provide mathematical models of many important problems in the physical sciences and engineering. This paper treats one class of such equations, concentrating on methods involving the use of classical fixed point theorem. The study of integral equations in connection with nonlinear equations has a long history, during which a variety of approaches has emerged. Here, we effectively use a strategy that derives key properties of the solvability of integral equations from previously established results in Hölder spaces. Moreover, our approach leads to solvability of the Fredholm integral equations.
\end{abstract}

2010 AMS Classification: 45B05, 45G10, 47H10

Keywords: Hölder condition, Fredholm integral equation, Schauder fixed point theorem.

\section{INTRODUCTION}

Fredholm integral equations show up thoroughly in many scientific areas like approximation theory, computational mathematics, physical mathematics and contact problems in the theory of elasticity. A short time since, several authors have comprehensively studied the integral equations and the solution of the Fredholm integral equation with the fixed point approach [1-10].

For example, J. Caballero, M. Darwish and K. Sadarangani et al. [3] study the following equation;

$$
x(t)=p(t)+x(t) \int_{0}^{1} k(t, \tau) x(r(\tau)) d \tau .
$$

This article concerns the entity of solutions of the a quadratic integral equation of Fredholm type,

$$
x(t)=\left(T_{1} x\right)(t)+\left(T_{2} x\right)(t) \int_{0}^{1} k(t, \tau) x(\rho(\tau)) d \tau, \quad t \in I=[0,1],
$$

where $k, \rho$ are given functions, $T_{1}, T_{2}$ are given operators satisfying conditions specified later and $x$ is unknown function. Notice that equation (1.1) in study [3] is a particular case of (1.2), for $\left(T_{1} x\right)(t)=p(t)$ and $\left(T_{2} x\right)(t)=x(t)$.

Email address: mervetemizer@hotmail.com (M.T. Ersoy) 


\section{Preliminaries}

Let $[a, b]$ be a closed interval in $\mathbb{R}$, by $C[a, b]$ we indicate the space of continuous functions defined on $[a, b]$ equipped with the supremum norm, i.e.,

$$
\|x\|_{\infty}=\sup \{|x(t)|: t \in[a, b]\}
$$

for $x \in C[a, b]$. For a fixed $\alpha$ with $0<\alpha \leq 1$, by $H_{\alpha}[a, b]$ we will indicate the spaces of the real functions $x$ defined on $[a, b]$ and satisfying the Hölder condition, that is, those functions $x$ for which there exists a constant $H_{x}^{\alpha}$ such that

$$
|x(t)-x(s)| \leq H_{x}^{\alpha}|t-s|^{\alpha}
$$

for all $t, s \in[a, b]$. It is well proved that $H_{\alpha}[a, b]$ is a linear subspaces of $C[a, b]$. Also, for $x \in H_{\alpha}[a, b]$, by $H_{x}^{\alpha}$ we will indicate the least possible stable for which inequality (2.1) is satisfied. Rather, we put

$$
H_{x}^{\alpha}=\sup \left\{\frac{|x(t)-x(s)|}{|t-s|^{\alpha}}: t, s \in[a, b] \text { and } t \neq s\right\} .
$$

The space $H_{\alpha}[a, b]$ with $0<\alpha \leq 1$ may be equipped with the norm

$$
\|x\|_{\alpha}=|x(a)|+H_{x}^{\alpha}
$$

for $x \in H_{\alpha}[a, b]$. Here, $H_{x}^{\alpha}$ is defined by (2.2). In [1], the authors demonstrated that $\left(H_{\alpha}[a, b],\|\cdot\|_{\alpha}\right)$ with $0<\alpha \leq 1$ is a Banach space.

Lemma 2.1 ( [1]). For $0<\alpha \leq 1$ and $x \in H_{\alpha}[a, b]$, we have:

$$
\|x\|_{\infty} \leq \max \left(1,(b-a)^{\alpha}\right)\|x\|_{\alpha} .
$$

In particular, the inequality $\|x\|_{\infty} \leq\|x\|_{\alpha}$ is satisfied for $a=0$ and $b=1$.

Lemma 2.2 ( [1]). For $0<\alpha<\beta \leq 1$, we have

$$
H_{\beta}[a, b] \subset H_{\alpha}[a, b] \subset C[a, b] .
$$

Furthermore, for $x \in H_{\beta}[a, b]$, we have:

$$
\|x\|_{\alpha} \leq \max \left(1,(b-a)^{\beta-\alpha}\right)\|x\|_{\beta} .
$$

Particularly, the inequality $\|x\|_{\infty} \leq\|x\|_{\alpha} \leq\|x\|_{\beta}$ is satisfied for $a=0$ and $b=1$.

Lemma 2.3 ( [3]). Let's assume that $0<\alpha<\beta \leq 1$ and $E$ is a bounded subset in $H_{\beta}[a, b]$, then $E$ is a relatively compact subset in $H_{\alpha}[a, b]$.

Lemma 2.4 ( [3]). Assume that $0<\alpha<\beta \leq 1$ and by $B_{r}^{\beta}$ we indicate the ball centered at $\theta$ and radius $r$ in the space $H_{\beta}[a, b]$, i.e., $B_{r}^{\beta}=\left\{x \in H_{\beta}[a, b]:\|x\|_{\beta} \leq r\right\}$. Then $B_{r}^{\beta}$ is a closed subset of $H_{\alpha}[a, b]$.

Corollary 2.5 ( [3]). Assume that $0<\alpha<\beta \leq 1$ and $B_{r}^{\beta}=\left\{x \in H_{\beta}[a, b]:\|x\|_{\beta} \leq r\right\}$. Then $B_{r}^{\beta}$ is a compact subset in the space $H_{\alpha}[a, b]$.

Theorem 2.6 (Schauder's fixed point theorem [10]). Let E be a nonempty and convex subset of a Banach space $(X,\|\cdot\|)$ and let $T: E \rightarrow E$ be a continuity mapping. Then $T$ has at least one fixed point in $E$.

\section{Main Result}

Theorem 3.1. Assume that the following conditions (i) - (iv) are satisfied:

(i) The operators $T_{1}, T_{2}: H_{\beta}[0,1] \rightarrow H_{\beta}[0,1]$ are continuous on $H_{\beta}[0,1]$ with respect to the norm $\|\cdot\|_{\alpha}$. Also, $T_{1}$ and $T_{2}$ hold the inequalities

$$
\left\|T_{1} x\right\|_{\beta} \leq f_{1}\left(\|x\|_{\beta}\right) \text { and }\left\|T_{2} x\right\|_{\beta} \leq f_{2}\left(\|x\|_{\beta}\right)
$$

for any $x \in H_{\beta}[0,1]$, where $\alpha$ and $\beta$ are the fixed constants satisfying $0<\alpha<\beta \leq 1$ and the functions $f_{1}, f_{2}: \mathbb{R}_{+} \rightarrow \mathbb{R}_{+}$are nondecreasing on $\mathbb{R}_{+}$.

(ii) $k:[0,1] \times[0,1] \rightarrow \mathbb{R}$ is a continuous function such that there exists a constant $k_{\beta}>0$ satisfying

$$
|k(t, \tau)-k(s, \tau)| \leq k_{\beta}|t-s|^{\beta},
$$

for any $t, s, \tau \in[0,1]$. 
(iii) $\rho:[0,1] \rightarrow[0,1]$ is measurable function.

(iv) There exists a positive solution $r_{0}$ of the inequality

$$
f_{1}(r)+\left(2 K+k_{\beta}\right) r f_{2}(r) \leq r,
$$

where the constant $K$ is defined by

$$
\sup \left\{\int_{0}^{1}|k(t, \tau)| d \tau: t \in[0,1]\right\} \leq K .
$$

Then the equation (1.2) has at least one solution $x=x(t)$ belonging to space $H_{\alpha}[0,1]$.

Proof. Let us consider $x \in H_{\beta}[0,1]$ and the operator $F$ defined on the space $H_{\beta}[0,1]$ by the formula:

$$
(F x)(t)=\left(T_{1} x\right)(t)+\left(T_{2} x\right)(t) \int_{0}^{1} k(t, \tau) x(\rho(\tau)) d \tau,
$$

for $t \in[0,1]$. Then for arbitrarily fixed $t, s \in[0,1],(t \neq s)$, in view of our assumptions we get

$$
\begin{aligned}
(F x)(t)-(F x)(s)= & \left(T_{1} x\right)(t)+\left(T_{2} x\right)(t) \int_{0}^{1} k(t, \tau) x(\rho(\tau)) d \tau \\
& -\left(T_{1} x\right)(s)-\left(T_{2} x\right)(s) \int_{0}^{1} k(s, \tau) x(\rho(\tau)) d \tau \\
= & \left(T_{1} x\right)(t)-\left(T_{1} x\right)(s)+\left(T_{2} x\right)(t) \int_{0}^{1} k(t, \tau) x(\rho(\tau)) d \tau \\
& -\left(T_{2} x\right)(s) \int_{0}^{1} k(s, \tau) x(\rho(\tau)) \\
& +\left(T_{2} x\right)(s) \int_{0}^{1} k(t, \tau) x(\rho(\tau)) d \tau \\
& -\left(T_{2} x\right)(s) \int_{0}^{1} k(t, \tau) x(\rho(\tau)) d \tau \\
= & \left(T_{1} x\right)(t)-\left(T_{1} x\right)(s)+\left(\left(T_{2} x\right)(t)-\left(T_{2} x\right)(s)\right) \int_{0}^{1} k(t, \tau) x(\rho(\tau)) d \tau \\
& +\left(T_{2} x\right)(s) \int_{0}^{1}(k(t, \tau)-k(s, \tau)) x(\rho(\tau)) d \tau
\end{aligned}
$$

and

$$
\begin{aligned}
\frac{|(F x)(t)-(F x)(s)|}{|t-s|^{\beta} \leq} & \frac{\left|\left(T_{1} x\right)(t)-\left(T_{1} x\right)(s)\right|}{|t-s|^{\beta}}+\frac{\left|\left(T_{2} x\right)(t)-\left(T_{2} x\right)(s)\right|}{\mid t-s^{\beta}} \int_{0}^{1}|k(t, \tau)||x(\rho(\tau))| d \tau \\
& +\frac{\left|\left(T_{2} x\right)(s)\right|}{|t-s|^{\beta}} \int_{0}^{1}|k(t, \tau)-k(s, \tau)||x(\rho(\tau))| d \tau \\
\leq & H_{T_{1} x}^{\beta}+\|x\|_{\infty}\left\|T_{2} x\right\|_{\beta} \int_{0}^{1}|k(t, \tau)| d \tau \\
& +\|x\|_{\infty}\left\|T_{2} x\right\|_{\infty} \int_{0}^{1} \frac{|k(t, \tau)-k(s, \tau)|}{|t-s|^{\beta}} d \tau \\
\leq & H_{T_{1} x}^{\beta}+\|x\|_{\beta}\left\|T_{2} x\right\|_{\beta} K+\|x\|_{\beta}\left\|T_{2} x\right\|_{\beta} \int_{0}^{1} k_{\beta} \frac{|t-s|^{\beta}}{|t-s|^{\beta}} d \tau \\
\leq & H_{T_{1} x}^{\beta}+\|x\|_{\beta} f_{2}\left(\|x\|_{\beta}\right) K+\|x\|_{\beta} f_{2}\left(\|x\|_{\beta}\right) k_{\beta} \\
= & H_{T_{1} x}^{\beta}+\left(K+k_{\beta}\right)\|x\|_{\beta} f_{2}\left(\|x\|_{\beta}\right) .
\end{aligned}
$$

This demonstrates that the operator $F$ maps $H_{\beta}[0,1]$ into itself. 
Besides, for any $x \in H_{\beta}[0,1]$, we get

$$
\begin{aligned}
|(F x)(0)| & \leq\left|\left(T_{1} x\right)(0)\right|+\left|\left(T_{2} x\right)(0)\right| \int_{0}^{1}|k(0, \tau)||x(\rho(\tau))| d \tau \\
& \leq\left|\left(T_{1} x\right)(0)\right|+\left\|T_{2} x\right\|_{\infty}\|x\|_{\infty} K \\
& \leq\left|\left(T_{1} x\right)(0)\right|+\left\|T_{2} x\right\|_{\beta}\|x\|_{\beta} K \\
& \leq\left|\left(T_{1} x\right)(0)\right|+\|x\|_{\beta} f_{2}\left(\|x\|_{\beta}\right) K .
\end{aligned}
$$

By the inequalities by (3.1) and (3.2), we derive that

$$
\begin{aligned}
\|F x\|_{\beta} & \leq\left\|T_{1} x\right\|_{\beta}+\left(2 K+k_{\beta}\right)\|x\|_{\beta} f_{2}\left(\|x\|_{\beta}\right) \\
& \leq f_{1}\left(\|x\|_{\beta}\right)+\left(2 K+k_{\beta}\right)\|x\|_{\beta} f_{2}\left(\|x\|_{\beta}\right) .
\end{aligned}
$$

Since positive number $r_{0}$ is the solution of the inequality given in hypothesis (iv), from (3.3), we conclude that the inequality

$$
\|F x\|_{\beta} \leq f_{1}\left(r_{0}\right)+\left(2 K+k_{\beta}\right) r_{0} f_{2}\left(r_{0}\right) \leq r_{0}
$$

holds. As a results, it follows from (3.4) that $F$ transforms the ball

$$
B_{r_{0}}^{\beta}=\left\{x \in H_{\beta}[0,1]:\|x\|_{\beta} \leq r_{0}\right\}
$$

into itself. That is, $F: B_{r_{0}}^{\beta} \rightarrow B_{r_{0}}^{\beta}$. Thus, we have that the set $B_{r_{0}}^{\beta}$ is relatively compact in $H_{\alpha}[0,1]$ for any $0<\alpha<\beta \leq 1$. Furthermore, $B_{r_{0}}^{\beta}$ is a compact subset in $H_{\alpha}[0,1]$.

We will show that the operator $F$ is continuous on $B_{r_{0}}^{\beta}$ with respect to the norm $\|\cdot\|_{\alpha}$, where $0<\alpha<\beta \leq 1$. Let $y \in B_{r_{0}}^{\beta}$ be an arbitrary point in $B_{r_{0}}^{\beta}$. Then, we get

$$
\begin{aligned}
(F x)(t)-(F y)(t)-((F x)(s)-(F y)(s))= & \left(T_{1} x\right)(t)+\left(T_{2} x\right)(t) \int_{0}^{1} k(t, \tau) x(\rho(\tau)) d \tau \\
& -\left(T_{1} y\right)(t)-\left(T_{2} y\right)(t) \int_{0}^{1} k(t, \tau) y(\rho(\tau)) d \tau \\
& -\left(T_{1} x\right)(s)-\left(T_{2} x\right)(s) \int_{0}^{1} k(s, \tau) x(\rho(\tau)) d \tau \\
& +\left(T_{1} y\right)(s)+\left(T_{2} y\right)(s) \int_{0}^{1} k(s, \tau) y(\rho(\tau)) d \tau
\end{aligned}
$$

for any $x \in B_{r_{0}}^{\beta}$ and $t, s \in[0,1]$. Let us take

$$
\left(T_{3} x\right)(\tau)=x(\rho(\tau)) \text { and }\left(T_{3} y\right)(\tau)=y(\rho(\tau))
$$

for the sake of shortness, then the equality (3.5) can be rewritten as:

$$
\begin{aligned}
(F x)(t)-(F y)(t)-((F x)(s)-(F y)(s))= & \left(T_{1} x\right)(t)-\left(T_{1} y\right)(t)-\left(\left(T_{1} x\right)(s)-\left(T_{1} y\right)(s)\right) \\
& +\left(T_{2} x\right)(t) \int_{0}^{1} k(t, \tau)\left(T_{3} x\right)(\tau) d \tau-\left(T_{2} y\right)(t) \int_{0}^{1} k(t, \tau)\left(T_{3} x\right)(\tau) d \tau \\
& +\left(T_{2} y\right)(t) \int_{0}^{1} k(t, \tau)\left(T_{3} x\right)(\tau) d \tau-\left(T_{2} y\right)(t) \int_{0}^{1} k(t, \tau)\left(T_{3} y\right)(\tau) d \tau \\
& -\left(T_{2} x\right)(s) \int_{0}^{1} k(s, \tau)\left(T_{3} x\right)(\tau) d \tau+\left(T_{2} y\right)(s) \int_{0}^{1} k(s, \tau)\left(T_{3} x\right)(\tau) d \tau \\
& -\left(T_{2} y\right)(s) \int_{0}^{1} k(s, \tau)\left(T_{3} x\right)(\tau) d \tau+\left(T_{2} y\right)(s) \int_{0}^{1} k(s, \tau)\left(T_{3} y\right)(\tau) d \tau .(3.6)
\end{aligned}
$$


By (3.6), we have

$$
\begin{aligned}
(F x)(t)-(F y)(t)-((F x)(s)-(F y)(s))= & \left(T_{1} x\right)(t)-\left(T_{1} y\right)(t)-\left(\left(T_{1} x\right)(s)-\left(T_{1} y\right)(s)\right) \\
& +\left(\left(T_{2} x\right)(t)-\left(T_{2} y\right)(t)\right) \int_{0}^{1} k(t, \tau)\left(T_{3} x\right)(\tau) d \tau \\
& +\left(T_{2} y\right)(t) \int_{0}^{1} k(t, \tau)\left(\left(T_{3} x\right)(\tau)-\left(T_{3} y\right)(\tau)\right) d \tau \\
& -\left(\left(T_{2} x\right)(s)-\left(T_{2} y\right)(s)\right) \int_{0}^{1} k(s, \tau)\left(T_{3} x\right)(\tau) d \tau \\
& -\left(T_{2} y\right)(s) \int_{0}^{1} k(s, \tau)\left(\left(T_{3} x\right)(\tau)-\left(T_{3} y\right)(\tau)\right) d \tau
\end{aligned}
$$

(3.7) yields the following equality:

$$
\begin{aligned}
((F x)(t)-(F y)(t))-((F x)(s)-(F y)(s))= & \left(T_{1} x\right)(t)-\left(T_{1} y\right)(t)-\left(\left(T_{1} x\right)(s)-\left(T_{1} y\right)(s)\right) \\
& +\left[\left(\left(T_{2} x\right)(t)-\left(T_{2} y\right)(t)\right)-\left(\left(T_{2} x\right)(s)-\left(T_{2} y\right)(s)\right)\right] \int_{0}^{1} k(t, \tau)\left(T_{3} x\right)(\tau) d \tau \\
& +\left(\left(T_{2} x\right)(s)-\left(T_{2} y\right)(s)\right) \int_{0}^{1}(k(t, \tau)-k(s, \tau))\left(T_{3} x\right)(\tau) d \tau \\
& +\left(\left(T_{2} y\right)(t)-\left(T_{2} y\right)(s)\right) \int_{0}^{1} k(t, \tau)\left(\left(T_{3} x\right)(\tau)-\left(T_{3} y\right)(\tau)\right) d \tau \\
& +\left(T_{2} y\right)(s) \int_{0}^{1}(k(t, \tau)-k(s, \tau))\left(\left(T_{3} x\right)(\tau)-\left(T_{3} y\right)(\tau)\right) d \tau .
\end{aligned}
$$

Since $\left|\left(T_{3} x\right)(\tau)\right| \leq\|x\|_{\infty}$ and $\left|\left(T_{3} x\right)(\tau)-\left(T_{3} y\right)(\tau)\right| \leq\|x-y\|_{\infty}$ for all $x, y \in B_{r_{0}}^{\beta}$ and $\tau \in[0,1]$, taking into account (3.8) and hypotheses, we can write:

$$
\begin{aligned}
\frac{|(F x)(t)-(F y)(t)-((F x)(s)-(F y)(s))|}{|t-s|^{\alpha}} & \frac{\left|\left(T_{1} x\right)(t)-\left(T_{1} y\right)(t)-\left(\left(T_{1} x\right)(s)-\left(T_{1} y\right)(s)\right)\right|}{|t-s|^{\alpha}} \\
& +\frac{\left|\left(T_{2} x\right)(t)-\left(T_{2} y\right)(t)-\left(\left(T_{2} x\right)(s)-\left(T_{2} y\right)(s)\right)\right|}{|t-s|^{\alpha}} \int_{0}^{1}\left|k(t, \tau) \|\left(T_{3} x\right)(\tau)\right| d \tau \\
& +\frac{\left|\left(T_{2} x\right)(s)-\left(T_{2} y\right)(s)\right|}{|t-s|^{\alpha}} \int_{0}^{1}\left|k(t, \tau)-k(s, \tau) \|\left(T_{3} x\right)(\tau)\right| d \tau \\
& +\frac{\left|\left(T_{2} y\right)(t)-\left(T_{2} y\right)(s)\right|}{|t-s|^{\alpha}} \int_{0}^{1}\left|k(t, \tau) \|\left(T_{3} x\right)(\tau)-\left(T_{3} y\right)(\tau)\right| d \tau \\
& +\frac{\left|\left(T_{2} y\right)(s)\right|}{|t-s|^{\alpha} \int_{0}^{1}\left|k(t, \tau)-k(s, \tau) \|\left(T_{3} x\right)(\tau)-\left(T_{3} y\right)(\tau)\right| d \tau} \\
\leq & \left\|T_{1} x-T_{1} y\right\|_{\alpha}+\left\|T_{2} x-T_{2} y\right\|_{\alpha}\|x\|_{\infty} K+\left\|T_{2} x-T_{2} y\right\|_{\infty}\|x\|_{\infty} \int_{0}^{1} k_{\beta}|t-s|^{\beta-\alpha} d \tau \\
& +\left\|T_{2} y\right\|_{\alpha}\|x-y\|_{\infty} K+\left\|T_{2} y\right\|_{\infty}\|x-y\|_{\infty} \int_{0}^{1} k_{\beta}|t-s|^{\beta-\alpha} d \tau \\
\leq & \left\|T_{1} x-T_{1} y\right\|_{\alpha}+K\left\|T_{2} x-T_{2} y\right\|_{\alpha}\|x\|_{\alpha}+k_{\beta}\left\|T_{2} x-T_{2} y\right\|_{\alpha}\|x\|_{\alpha} \\
& +K\left\|T_{2} y\right\|_{\alpha}\|x-y\|_{\alpha}+k_{\beta}\left\|T_{2} y\right\|_{\alpha}\|x-y\|_{\alpha} \\
= & \left\|T_{1} x-T_{1} y\right\|_{\alpha}+\left(K+k_{\beta}\right)\left\|T_{2} x-T_{2} y\right\|_{\alpha}\|x\|_{\alpha}+\left(K+k_{\beta}\right)\left\|T_{2} y\right\|_{\alpha}\|x-y\|_{\alpha}
\end{aligned}
$$


for all $t, s \in[0,1]$ with $t \neq s$. Besides, for $x, y \in B_{r_{0}}^{\beta}$, we obtain following equality:

$$
\begin{aligned}
(F x)(0)-(F y)(0)= & \left(T_{1} x\right)(0)+\left(T_{2} x\right)(0) \int_{0}^{1} k(0, \tau)\left(T_{3} x\right)(\tau) d \tau \\
& -\left(T_{1} y\right)(0)-\left(T_{2} y\right)(0) \int_{0}^{1} k(0, \tau)\left(T_{3} y\right)(\tau) d \tau \\
= & \left(T_{1} x\right)(0)-\left(T_{1} y\right)(0)+\left(T_{2} x\right)(0) \int_{0}^{1} k(0, \tau)\left(T_{3} x\right)(\tau) d \tau \\
& -\left(T_{2} y\right)(0) \int_{0}^{1} k(0, \tau)\left(T_{3} x\right)(\tau) d \tau+\left(T_{2} y\right)(0) \int_{0}^{1} k(0, \tau)\left(T_{3} x\right)(\tau) d \tau \\
& -\left(T_{2} y\right)(0) \int_{0}^{1} k(0, \tau)\left(T_{3} y\right)(\tau) d \tau \\
= & \left(T_{1} x\right)(0)-\left(T_{1} y\right)(0)+\left(\left(T_{2} x\right)(0)-\left(T_{2} y\right)(0)\right) \int_{0}^{1} k(0, \tau)\left(T_{3} x\right)(\tau) d \tau \\
& +\left(T_{2} y\right)(0) \int_{0}^{1} k(0, \tau)\left(\left(T_{3} x\right)(\tau)-\left(T_{3} y\right)(\tau)\right) d \tau .
\end{aligned}
$$

By (3.10), we get that

$$
\begin{aligned}
|(F x)(0)-(F y)(0)| \leq & \left|\left(T_{1} x\right)(0)-\left(T_{1} y\right)(0)\right|+\left|\left(T_{2} x\right)(0)-\left(T_{2} y\right)(0)\right||k(0, \tau)| \int_{0}^{1}\left|\left(T_{3} x\right)(\tau)\right| d \tau \\
& +\left|\left(T_{2} y\right)(0)\right||k(0, \tau)| \int_{0}^{1}\left|\left(T_{3} x\right)(\tau)-\left(T_{3} y\right)(\tau)\right| d \tau \\
\leq & \left\|T_{1} x-T_{1} y\right\|_{\infty}+\left\|T_{2} x-T_{2} y\right\|_{\infty} K\|x\|_{\infty}+\left\|T_{2} y\right\|_{\infty} K\|x-y\|_{\infty} \\
\leq & \left\|T_{1} x-T_{1} y\right\|_{\alpha}+\left\|T_{2} x-T_{2} y\right\|_{\alpha} K\|x\|_{\alpha}+\left\|T_{2} y\right\|_{\alpha} K\|x-y\|_{\alpha} .
\end{aligned}
$$

From (3.9) and (3.11), we have that

$$
\begin{aligned}
\|F x-F y\|_{\alpha}= & |(F x-F y)(0)|+H_{F x-F y}^{\alpha} \\
= & |(F x)(0)-(F y)(0)| \\
& +\sup \left\{\frac{|(F x)(t)-(F y)(t)-((F x)(s)-(F y)(s))|}{|t-s|^{\alpha}}: t, s \in[0,1] \text { and } t \neq s\right\} \\
\leq & 2\left\|T_{1} x-T_{1} y\right\|_{\alpha}+\left(2 K+k_{\beta}\right)\left\|T_{2} x-T_{2} y\right\|_{\alpha}\|x\|_{\alpha} \\
& +\left(2 K+k_{\beta}\right)\left\|T_{2} y\right\|_{\alpha}\|x-y\|_{\alpha} \\
\leq & 2\left\|T_{1} x-T_{1} y\right\|_{\alpha}+\left(2 K+k_{\beta}\right)\left\|T_{2} x-T_{2} y\right\|_{\alpha}\|x\|_{\beta} \\
& +\left(2 K+k_{\beta}\right)\left\|T_{2} y\right\|_{\beta}\|x-y\|_{\alpha} \\
\leq & 2\left\|T_{1} x-T_{1} y\right\|_{\alpha}+\left(2 K+k_{\beta}\right)\left\|T_{2} x-T_{2} y\right\|_{\alpha}\|x\|_{\beta} \\
& +\left(2 K+k_{\beta}\right) f_{2}\left(\|y\|_{\beta}\right)\|x-y\|_{\alpha} .
\end{aligned}
$$

Moreover, since $\|x\|_{\beta} \leq r_{0}$ and $\|y\|_{\beta} \leq r_{0}$, we derive from (3.12) that the following inequality holds:

$$
\begin{aligned}
\|F x-F y\|_{\alpha} \leq & 2\left\|T_{1} x-T_{1} y\right\|_{\alpha}+\left(2 K+k_{\beta}\right) r_{0}\left\|T_{2} x-T_{2} y\right\|_{\alpha} \\
& +\left(2 K+k_{\beta}\right) f_{2}\left(r_{0}\right)\|x-y\|_{\alpha} .
\end{aligned}
$$

Since the operators $T_{1}, T_{2}: H_{\beta}[0,1] \rightarrow H_{\beta}[0,1]$ are continuous on $H_{\beta}[0,1]$ with respect to the norm $\|\cdot\|_{\alpha}$, they are also continuous at the point $y \in B_{r_{0}}^{\beta}$. Let us take an arbitrary $\varepsilon>0$, then there exists the number $\delta$ satisfying

$$
0<\delta<\frac{\varepsilon}{3\left(2 K+k_{\beta}\right) f_{2}\left(r_{0}\right)}
$$

such that the inequalities

$$
\left\|T_{1} x-T_{1} y\right\|_{\alpha}<\frac{\varepsilon}{6}
$$


and

$$
\left\|T_{2} x-T_{2} y\right\|_{\alpha}<\frac{\varepsilon}{3\left(2 K+k_{\beta}\right) r_{0}}
$$

hold for all $x \in B_{r_{0}}^{\beta}$, where $\|x-y\|_{\alpha}<\delta$. Then, taking into account (3.13), we derive the following inequality:

$$
\|F x-F y\|_{\alpha}<\varepsilon
$$

for all $x \in B_{r_{0}}^{\beta}$ with $\|x-y\|_{\alpha}<\delta$. As a results, we infer that the operator $F$ is continuous at the point $y \in B_{r_{0}}^{\beta}$. Because $y$ was chosen arbitrarily, we deduce that $F$ is continuous on $B_{r_{0}}^{\beta}$ with respect to the norm $\|\cdot\|_{\alpha}$. As $B_{r_{0}}^{\beta}$ is compact in $H_{\alpha}[0,1]$, from the classical Schauder fixed point theorem, we get the desired result.

\section{CONFLICTS OF INTEREST}

The author declares that there are no conflicts of interest regarding the publication of this article.

\section{REFERENCES}

[1] Banaś, J., Nalepa, R., On the space of functions with growths tempered by a modulus of continuity and its applications, J. Funct. Space Appl., (2013), 13 pages. doi:10.1155/2013/820437

[2] Berenguer, M.I., Munoz, M.V.F., Guillem, A.I.G., Galan, M.R., Numerical treatment of fixed point applied to the nonlinear Fredholm integral equation, Fixed Point Theory Appl., 2009(2009), 1-8.

[3] Caballero, J., Abdalla, M., Sadarangani, K., Solvability of a quadratic integral equation of fredholm type in Hölder spaces, Electron. J. Differ. Eq., 31(2014), 1-10.

[4] Caballero, M.J., Nalepa, R., Sadarangani, K., Solvability of a quadratic integral equation of Fredholm type with supremum in Hölder spaces, J. Funct. Space Appl., (2014).

[5] Fredholm, E.I., Sur une classe d'équations fonctionnelles, Acta Math., 27(1903), 365-390.

[6] Kirk, W.A., Srinavasan, P.S., Veeramani, P., Fixed Points for mapping satisfying cyclical contractive conditions, Fixed Point Theory, 4(2003), 79-89.

[7] Pathak, H.K., Khan, M.S., Tiwari, R., A common fixed point theorem and its application to nonlinear integral equations, Comput. Math. Appl. Arch., 53(2007), 961-71.

[8] Rasham, T., Shoaib, A., Hussain, N., Arshad, M., Khan, S.U., Common fixed point results for new Ciric-type rational multivalued F-contraction with an application, J. Fixed Point Theory Appl., 20(1)(2018), 1-16.

[9] Rus, M.D., A note on the existaence of positive solution of Fredholm integral equations, Fixed Point Theory, 5(2004), 369-377.

[10] Schauder, J., Der Fixpunktsatz in Funktionalriiumen, Studia Math., 2(1930), 171-180. 